Artigo Original

\title{
ANÁLISE FÍSICO-QUÍMICA E MICROBIOLÓGICA DE SISTEMAS DE PRODUÇÃO E DISTRIBUIÇÃO DE AR RESPIRÁVEL DE USO HOSPITALAR COM PROPOSTA DE MELHORIA
}

\author{
Autor: Roberto da Silva Gusmão1, Francisco Sandro Menezes Rodrigues', Denise Freitas', \\ Auro Rodrigues Wolff' ${ }^{2}$, Giovani Ribeiro ${ }^{3}$, Raul Diniz Ragazzi ${ }^{4}$, Ogier Santana ${ }^{5}$. \\ 1Universidade Federal de São Paulo. ${ }^{2}$ Milliespress. ${ }^{3}$ Donaldson. ${ }^{4}$ Ophthalmos. ${ }^{5} \mathrm{OGS}$ ambiental
}

\section{Informações do artigo}

Palavras Chave:

Microrganismos

transportados pelo ar;

Hospital; Qualidade do ar

interno, Ar comprimido;

Saúde pública; Utilidade pública.

\section{Resumo}

A qualidade do ar respirável (AR) utilizado no manejo de paciente tem sido objeto de interesse em medicina, uma vez que, se não adequada, pode oferecer o risco de doenças de várias etiologias, incluindo as de caráter infeccioso. Os sistemas de produção, armazenamento e distribuição do AR medicinal devem garantir condições seguras de funcionamento para uma efetiva manutenção da saúde. Há normas que regem e definem valores de segurança para o ar respirável medicinal utilizado em centros de atendimento à saúde. $\mathrm{O}$ objetivo deste trabalho foi analisar o ar respirável em centro cirúrgico oftalmológico com envolvimento do seu preparo, distribuição e entrega no ponto de uso.

\section{Article ID}

Keywords:

Airborne microorganisms;

Hospital; Indoor air quality; Compressed air; Public health; Public utility.

\begin{abstract}
The quality of breathable air (RA) used in patient management has been a subject of interest in medicine, as if not adequate, it may present the risk of diseases of various etiologies, including infectious ones. Medical RA production, storage and distribution systems must ensure safe operating conditions for effective health maintenance. There are standards governing and setting safety values for medical breathing air used in healthcare facilities. The objective of this study was to analyze breathable air in an ophthalmic operating room with involvement of its preparation, distribution and delivery at the point of use.
\end{abstract}

\footnotetext{
${ }^{1}$ Autor correspondente:
}

Roberto da Silva Gusmão - e-mail: farmaceutico.gusmao@gmail.com - https://orcid.org/0000.0001.5546.2366

DOI: https://doi.org/10.31415/bjns.v2i3.68 - Artigo recebido em: 26 de junho de 2019; aceito em 05 de Julho de 2019 ; publicado em 30 de setembro de 2019. Brazilian Journal of Natural Sciences, Vol. 2, N.3, setembro 2019. Disponível online a partir de 30 de setembro de 2019, ISSN 2595-0584. www.bjns.com.br. Todos os autores contribuíram igualmente com o artigo. Os autores declaram não haver conflito de interesse. Este é um artigo de acesso aberto sob a licença CC - BY: http://creativecommons.org/licenses/by/4.0 


\section{Introdução}

A qualidade do ar respirável (AR) tem sido objeto de interesse em medicina, uma vez que é uma importante ferramenta utilizada em hospitais, centros cirúrgicos e clínicas, não só na ventilação mecânica ou inalação, mas também na exposição geral. Se não adequada, pode aumentar o risco de doenças de várias etiologias, incluindo as de caráter infecciosos. Os sistemas de produção, armazenamento e distribuição do AR devem garantir condições seguras de funcionamento para uma efetiva manutenção da saúde. Estas condições são particularmente importantes quando o foco é o AR de uso hospitalar. O AR de uso hospitalar é produzido por meio da captação do ar ambiente e comprimido por um compressor de ar, após a primeira etapa que é a de captação e compressão o AR passa por processo de filtração e separação de substâncias, secagem, armazenamento e distribuição. Dependendo de como o projeto de instalação, operação e performance acontecer o sistema poderá ou não oferecer riscos. Para tanto, o local onde o compressor e ou sistema de produção estiverem instalados apresentarão altos ou baixos teores de gazes $\left(\mathrm{CO}^{2}, \mathrm{CO}\right.$, $\mathrm{SO}^{2}, \mathrm{NO}^{2}$ ), partículas que transportam contaminantes microbiológicos, ponto de orvalho (capacidade do vapor de água passar para o estado líquido em condições de temperatura e pressão) exacerbado e vapor de água elevado. $\mathrm{O}$ mal funcionamento de um sistema de produção e distribuição de AR pode acontecer por vários motivos, dentre os motivos a falta de limpeza e manutenção preventiva programada na linha de distribuição, ausência troca de filtros dentro do prazo estabelecido pelo fabricante e qualificações periódicas de funcionamento no que se refere a teste físico-químicos e microbiológicos. Quanto às qualificações de performance, essas devem acontecer concomitantemente com as verificações tanto dos pontos de uso como de pontos de amostragem definidos pela empresa qualificadora do sistema. Isso deve ser feito para entender como o sistema de maneira global está se comportando e quais os possíveis desvios que o sistema apresenta na qualidade do ar respirável entregue no ponto de uso. Pensando em qualificação devemos levar em consideração também alguns pontos que são primordiais para o mapeamento físico-químico e microbiológico de um sistema de produção de AR. Sendo assim, podemos elencar 6 pontos de amostragem: 1. após a geração de ar comprimido; 2 . no pulmão de armazenamento; 3. após o pulmão de armazenamento de ar; 4. após a secagem do ar; 5. após o banco de filtros; 6. no ponto de uso. Esses são alguns dos pontos de configuração importante quando se pensa em qualificação de um sistema de geração de ar respirável. Vale lembrar que nem todos os sistemas de geração de ar respirável possuem os mesmos itens apresentados na figura 01. No entanto, o princípio geral se resume basicamente nos pontos definidos pela OGS Ambiental, empresa que realiza qualificação de sistema de geração e distribuição de ar respirável comprimido.

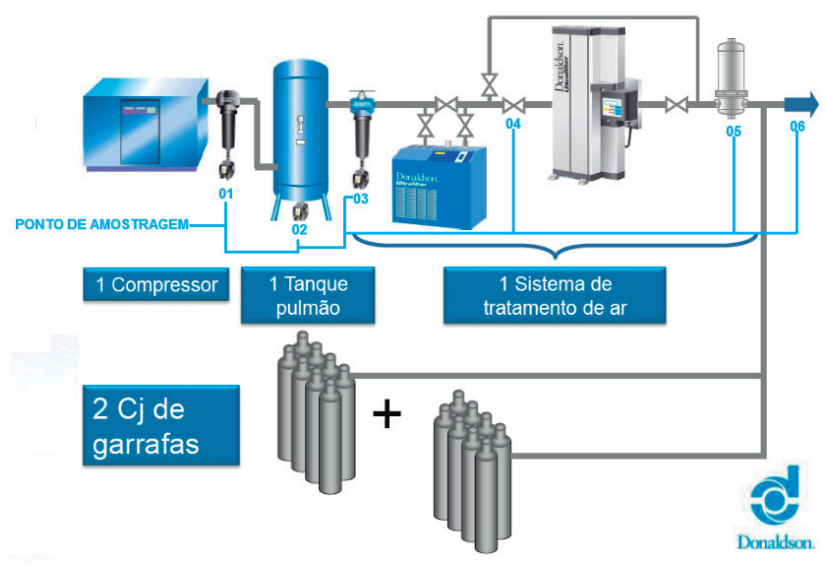

Fonte: Donaldson - https://ir.donaldson.com

Com base nos valores estabelecidos nas normas descritas acima, foi proposto neste estudo a coleta de amostras de AR em três diferentes pontos do departamento de oftalmologia e ciências visuais sediado na Universidade Federal de São Paulo. Os pontos escolhidos foram: 1. Abrigo de gases; 2. Centro cirúrgico oftalmol.; 3. Casa de máquinas.

\section{Material e Método}

Analisou-se parâmetros físico-químicos, microbiológicos e de contagem de partículas no sistema de produção de ar respirável que envolve o Centro Cirúrgico Ambulatorial (CCOFTALMO) do Departamento de Oftalmologia e Ciências Visuais da Escola Paulista de Medicina, Hospital São Paulo, Universidade Federal de São Paulo (UNIFESP).

Para a análise físico-química e de contagem de partículas este estudo teve apoio das empresas: Milliexpress Ind. Com. e Representações Ltda. (R. Bela Aliança, 75 - Vila Leopoldina, São Paulo - SP, 05088-030) e Donaldson do Brasil (Rod Dom Pedro I, S/N Km 93,6 - Emp. Zimba D. Pedro, CEP: 13.252- 
800, - Sitio da Moenda Itatiba / SP - Brasil). Para a avaliação microbiológica contou-se com o apoio da empresa OGS Ambiental Ltda. (Rua Conde Prates, 805 - Parque da Mooca, São Paulo - SP, 03122-000), líderes comerciais no assunto.

Para os testes físico-químicos utilizou-se conectores com engate rápido marca FESTO $6 \mathrm{~mm}$ e $8 \mathrm{~mm}$ (Festo Brasil, R. Giusepe Cresppi, 76 - Jd. Santa Emília, São Paulo, S.P.) e o aparelho Dew Point Meter, modelo DDM, (Donaldson do Brasil, Rodovia Dom Pedro I, S/N Km 93.6, Itatiba, S.P.) para avaliar o controle de ponto de orvalho (obtido no momento da amostragem logo após a coleta), umidade e temperatura da linha de distribuição de ar respirável. Como o sistema possui um banco de filtros para garantir a qualidade do ar respirável utilizado no local, foi proposta a coleta de ar no ponto de uso com e sem filtração terminal.

Para a contagem de partículas não viáveis utilizou-se o sistema contador de partículas Lasair III (Particle Measuring Systems Inc., 5475 Airport Blvd, Boulder, CO 80301, EUA).

Para os testes microbiológicos pesquisou-se a presença de bactérias e fungos utilizando o Amostrador de Ar Microbiológico Remoto MiniCapt ${ }^{\circledR}$ (Particle Measuring Systems Inc., 5475 Airport Blvd, Boulder, CO 80301, EUA), realizando-se a sedimentação de partículas amostradas em Agar Triptona de Soja (TSA), para cultivo de bactérias, e Agar Sabouraud para o cultivo de fungos. As amostras foram incubadas em estufas a $22,5^{\circ} \mathrm{C}+/-2,5^{\circ} \mathrm{C}$ para estimulação de crescimento de fungos e $32,5^{\circ} \mathrm{C}+/-2,5^{\circ} \mathrm{C}$ para estimulação de crescimento de bactérias. No caso das amostras para identificação de fungos as amostras ficaram incubadas durante 07 dias corridos a partir do dia da amostragem e as amostras para identificação de bactérias ficaram incubadas durante 03 dias.

Nos três pontos foram colhidos: 1 . Abrigo de gases: realizadas contagens de partículas viáveis e não viáveis; 2. Centro cirúrgico oftalmol.: realizados testes físico-químicos, contagens de partículas viáveis e não viáveis e testes microbiológicos; 3 . Casa de máquinas: realizados testes físico-químicos e contagens de partículas viáveis e não viáveis.

O filtro e a carcaça utilizada no estudo foram cedidos pela empresa Donaldison ${ }^{\circledR}$ do Brasil em parceria com a empresa MilliExpress. O aparato de teste foi utilizado no estudo esteve em caráter de empréstimo que serviu para mapear os desvios apresentados no sistema de geração de AR.
As coletas e contagens de partículas viáveis e não viáveis foram realizadas pela empresa OGS Ambiental que gerou um relatório técnico do estudo realizado no sistema de AR. As amostras microbiológicas foram incubadas no laboratório da mesma empresa durante o período de incubação tanto para fungos (7 dias de incubação) como para bactérias (3 dias de incubação).

\section{Resultados}

Em todos os pontos avaliados os parâmetros físico-químicos e de contagem de partículas não viáveis estavam dentro dos padrões de normalidade. Notou-se crescimento de $03 \mathrm{UFC} / \mathrm{cm}^{3}$ de bactérias e de 08 $\mathrm{UFC} / \mathrm{cm}^{3}$ de bolores e leveduras no ponto de abrigo de gases. Crescimento de bactérias (1 UFC/m3) no ponto centro cirúrgico oftalmológico, quando não se utilizou o filtro de proteção terminal, não havendo qualquer crescimento após a utilização do filtro. No que se refere à contagem de partículas não viáveis a quantidade de partículas contadas foi igual entre a amostra feita com o filtro terminal e sem o filtro terminal. Observou-se a presença de crescimento bacteriano de $01 \mathrm{UFC} / \mathrm{cm}^{3}$ no teste realizado sem o filtro terminal no momento da amostragem, resultados demonstrados na Tabela $\mathbf{1}$.

\section{Considerações / intervenções}

Considerando o crescimento de $3 \mathrm{UFC} / \mathrm{cm}^{3}$ colônias de bactérias e $8 \mathrm{UFC} / \mathrm{cm}^{3}$ colônias de fungos na sala de abrigo de gases, e de $1 \mathrm{UFC} / \mathrm{cm}^{3}$ colônia de bactérias e nenhuma colônia para fungos no ponto denominado centro cirúrgico oftalmológico, após passar por todas as fases de tratamento do AR, sugere-se que as bactérias estão rompendo a barreira feita pelos estágios de filtração ou no momento das instalações das tubulações, algumas decisões de montagem podem ter sido feita de forma inadequadamente não seguindo padrões de qualidade na montagem de sistemas de tratamento de ar respirável.

Com base na presença de $1 \mathrm{UFC} / \mathrm{cm}^{3}$ presente na linha de AR foi proposta de maneira preventiva a instalação de filtros terminais cuja malha deve ser de $0,22 \mu \mathrm{m}$ para servir de barreira microbiológica e entregar o AR isento de qualquer possibilidade de contaminação. 
Tabela 1.

\begin{tabular}{|c|c|c|c|c|c|}
\hline \multicolumn{2}{|l|}{ Parâmetros avaliados } & \multirow{2}{*}{$\begin{array}{l}\begin{array}{c}\text { Abrigo de } \\
\text { gases }\end{array} \\
\text { Resultados }\end{array}$} & \multirow{2}{*}{$\begin{array}{c}\text { Centro } \\
\text { cirúrgico } \\
\text { oftalmológico } \\
\text { Resultados }\end{array}$} & \multirow{2}{*}{$\begin{array}{c}\begin{array}{c}\text { Casa de } \\
\text { máquinas }\end{array} \\
\text { Resultados }\end{array}$} & \multirow[t]{2}{*}{ Critérios } \\
\hline & & & & & \\
\hline \multirow{2}{*}{$\begin{array}{l}\text { Contagem de partículas não } \\
\text { viáveis }\end{array}$} & $0,5 \mathrm{~mm}$ & $281.252 \mathrm{part} / \mathrm{m}^{3}$ & $241.447 \mathrm{part} / \mathrm{m}^{3}$ & $490.788 \mathrm{part} / \mathrm{m}^{3}$ & $3.520 .000 \mathrm{part} / \mathrm{m}^{3}$ \\
\hline & $5,0 \mathrm{~mm}$ & $4.657 \mathrm{part} / \mathrm{m}^{3}$ & $2.503 \mathrm{part} / \mathrm{m}^{3}$ & $926 \mathrm{part} / \mathrm{m}^{3}$ & $29.000 \mathrm{part} / \mathrm{m}^{3}$ \\
\hline \multirow{2}{*}{$\begin{array}{l}\text { Testes microbiológicos } \\
\text { (sem filtro) }\end{array}$} & Bactérias & $3 \mathrm{UFC} / \mathrm{m}^{3}$ & $1 \mathrm{UFC} / \mathrm{m}^{3}$ & NA & $0 \mathrm{UFC} / \mathrm{m}^{3}$ \\
\hline & Fungos & $8 \mathrm{UFC} / \mathrm{m}^{3}$ & $0 \mathrm{UFC} / \mathrm{m}^{3}$ & NA & $0 \mathrm{UFC} / \mathrm{m}^{3}$ \\
\hline \multirow{2}{*}{$\begin{array}{l}\text { Testes microbiológicos } \\
\text { (com filtro) }\end{array}$} & Bactérias & $0 \mathrm{UFC} / \mathrm{m}^{3}$ & $0 \mathrm{UFC} / \mathrm{m}^{3}$ & NA & $0 \mathrm{UFC} / \mathrm{m}^{3}$ \\
\hline & Fungos & $0 \mathrm{UFC} / \mathrm{m}^{3}$ & $0 \mathrm{UFC} / \mathrm{m}^{3}$ & NA & $0 \mathrm{UFC} / \mathrm{m}^{3}$ \\
\hline \multicolumn{2}{|l|}{ Teor de óleo } & NA & $(-)$ & $0,08 \mathrm{mg} / \mathrm{m}^{3}$ & $<0,1 \mathrm{mg} / \mathrm{m}^{3}$ \\
\hline \multicolumn{2}{|l|}{ Teor de oxigênio } & NA & $21,3 \%$ & $20,6 \%$ & $\begin{array}{c}19,5 \% \leq \mathrm{O}_{2} \leq \\
23,5 \%\end{array}$ \\
\hline \multicolumn{2}{|l|}{ Teor de monóxido de carbono } & NA & $<5 \mathrm{ppm}$ & $<5 \mathrm{ppm}$ & $\leq 5 \mathrm{ppm}$ \\
\hline \multicolumn{2}{|l|}{ Teor de dióxido de carbono } & NA & $89 \mathrm{ppm}$ & $87 \mathrm{ppm}$ & $\leq 500 \mathrm{ppm}$ \\
\hline
\end{tabular}

Tabela 1. resultados dos pontos do AR avaliados quanto aos parâmetros físico-químicos, de contagem de partículas viáveis e não viáveis Centro cirúrgico oftalmologia; part, partículas; UFC, unidades formadoras de colônias; NA, não avaliado - local impróprio para essa análise; ppm, partes por milhão.

Tabela 2.

\begin{tabular}{|l|c|c|c|c|}
\hline Testes realizados & Padrões estabelecidos em norma & Abrigo de gases & Casa de máquinas & $\begin{array}{c}\text { Centro cirúrgico } \\
\text { oftalmológico }\end{array}$ \\
\hline Dew Point & $-45,5 \mathrm{DP}$ & $-77,6 \mathrm{DP}$ & $-76,6 \mathrm{DP}$ & $-77,8 \mathrm{DP}$ \\
\hline Temperatura $^{\circ} \mathrm{C}$ & ----- & $20.5^{\circ} \mathrm{C}$ & $20.2^{\circ} \mathrm{C}$ & $22.1^{\circ} \mathrm{C}$ \\
\hline Umidade U\% $^{\circ}$ & ----- & $0,0 \%$ & $0,0 \%$ & $0,0 \%$ \\
\hline
\end{tabular}

Tabela 2. amostras e locais de amostragem realizados no dia da coleta.

Outra medida preventiva para a eliminação dos desvios de qualidade do AR é a realização da limpeza da linha e troca periódica do banco de filtros que compõem o sistema. Ainda não é mandatório para hospitais, clínicas e centros cirúrgicos mais se trata de uma medida preventiva realizar o teste de integridade de filtros periodicamente para se saber se a pressão da linha não rompeu a malha do filtro durante o período de uso. O rompimento da malha do filtro favorece a transferência de microrganismos e sujidades para o lado mais limpo da linha.

O ponto de orvalho coletado tanto no abrigo de gases como no centro cirúrgico oftalmológico apresentou resultados dentro do estabelecidos em normas. Foram verificados nesses pontos umidade, temperatura e ponto de orvalho, dados demonstrados na tabela 2 .

\section{Conclusão}

Pode se concluir que os pontos abrigo de gases e centro cirúrgico oftalmológico, por apresentarem crescimento microbiológicos, mesmo que em conformidades com a normas atuais de qualidade, merecem uma atenção especial. A distância entre o local de produção do ar respirável e o local de uso, por não existem pontos de drenagem e limpeza na linha de distribuição geram bolsões de água que podem favorecer a contaminação da linha. Sugere-se a instalação de filtros terminais de $0,22 \mu \mathrm{m}$ nos pontos de uso do AR terminalmente para a retenção em massa de possíveis microrganismos. Torna se importante realizar amostragens de ar no ponto de produção, distribuição e entrega do ar respirável periodicamente para se evitar a contaminação e monitorar as condições de 
funcionamento do sistema utilizado. É importante que a comunidade oftalmológica em geral se inteire do assunto e passe a questionar o sistema que utiliza no cuidado de seus pacientes.

\section{Referências utilizadas como base}

1. ASSOCIAÇÃO BRASILEIRA DE NORMAS TÉCNICAS. NBR 12.188: Sistemas centralizados de oxigênio, ar, óxido nitroso e vácuo para uso medicinal em estabelecimentos assistenciais de saúde: elaboração. Rio de Janeiro, 2003. 25p.

2. ASSOCIAÇÃO BRASILEIRA DE NORMAS TÉCNICAS. NBR 15.543: Equipamento de proteção respiratória: elaboração. Rio de Janeiro, 1999. 45p.

3. BIER, O. G. Microbiologia e Imunologia. 3. ed. São Paulo: Melhoramentos, 1984.

4. GHANIZADEH, F. and H. GODINI, A review of the chemical and biological pollutants in indoor air hospitals and assessing their effects on the health of patients, staff and visitors. Ver Environ Helth, 2018. 33 (3): p. 231-245.

5. INTERNATIONAL STANDARD ORGANIZATION. ISO 8573-1: Contaminants and purity classes: elaboração. Geneva, 2010. 16p.

6. INTERNATIONAL STANDARD ORGANIZATION. ISO 8573-2: Test methods for oil aerosol content: elaboração. Geneva, 2007. 30p.

7. LACAZ, C. da S., PORTO, E., HENS-VACCARI, E. M. and MELO, N. T. Guia para identificação de fungos actinomicetos e algas de interesse medico, $8^{\text {a }}$., ed. Sarvier, São Paulo, 1998.

8. N.F.P.A., Standard on gas and vacum systems, in NFPA 99C, NFPA. Editor, 2005: USA.

9. ODONTO-MÉDICO-HOSPITALAR, C. Sistemas centralizados de suprimento de gases medicinais, de gases para dispositivos médicos e de vácuo para uso em serviços de saúde, A.A.B.D.N. Técnicas, Editor, 2016.

10. ODONTO-MÉDICO-HOSPITALAR, C.B., Sistemas centralizados de oxigênio, ar, óxido nitroso e vácuo para uso medicinal em estabelecimentos assistenciais de saúde, in NBR 12.188, A.A.B.D.N. Técnicas, Editor, 2003: Rio de Janeiro.

11. PELCZAR Jr., M. J.; CHAN, E. C. S.; KRIEG, N. R. Microbiologia: conceitos e aplicações. V. 2. São Paulo: Makron Books, 1997.

12. SMIELOWSK, M., M. MARC, and B. ZABIEGALA, Indor air quality in public utility enveriro- menments a review. Environ Sci Pollut Res Int, 2017. 24 (12): p. 11176.

13. TRABULSI, L. R.; TOLEDO, M. R. F. de. Microbiologia. 2. ed. São Paulo: Atheneu, 1996. 99. 